\title{
Event Based Fairness for Video Surveillance Sensor Networks ${ }^{\star}$ (Work in Progress)
}

\author{
Yunus Durmus, Bahri Atay Ozgovde, and Cem Ersoy \\ Computer Networks Research Laboratory \\ Department of Computer Engineering \\ Boğaziçi University \\ Bebek 34342 Istanbul, Turkey \\ \{yunus.durmus1, ozgovde, ersoy\}@boun.edu.tr
}

\begin{abstract}
With their ease of installation, infrastructureless mode of operation and flexible deployment style, Video Surveillance Sensor Networks (VSSNs) provide more opportunities than legacy surveillance methods for applications such as habitat monitoring and border surveillance. We argue that events created in the coverage area of a VSSN are the application level messaging units and propose to employ Event Based Fairness (EBF) which aims at a fair distribution of nodes' resources according to the event flows. We carried out simulation experiments to compare the application level performances of two different EBF implementations with that of FCFS based queueing. We observe that EBF enhances the VSSN performance in two ways: Firstly, when the video traffic due to the events created exceed the total capacity of the network, EBF increases the overall number of events properly reported to the sink. Secondly, EBF reduces the initial event reporting delay, thus decreases the response time to the occurring events within the network.
\end{abstract}

Keywords: Video Surveillance Sensor Networks, Fairness, Queue Management.

\section{Introduction}

When compared with legacy Wireless Sensor Networks (WSNs) that operate on scalar data such as humidity and temperature, the visual information provided by Multimedia Wireless Sensor Networks (MWSNs) in general and Video Surveillance Sensor Networks (VSSNs) in particular, increase the accuracy of event identification and decrease the false alarm rate considerably. However, this enhanced identification capability comes with the additional complexity of increased traffic volume that needs to be processed according to the realtime QoS requirements. This is very challenging since, in spite of the increased application and networking level complexity, VSSNs are typically implemented on similar hardware designed for scalar WSNs [12].

\footnotetext{
* This work is supported by TUBITAK under the grant number 108E207.
} 


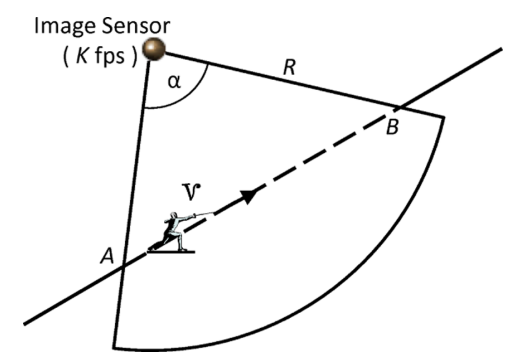

Fig. 1. Number of frames $F$ produced by a single sensor node upon detection is $F=$ $K \frac{D_{A B}}{V}$ where $K, V$ and $D_{A B}$ stands for the camera frame rate, target speed and pathlength respectively. The pathlength $D_{A B}$, in turn, depends on the sensing radius $R$ and the FoV, $\alpha$.

A VSSN operates in event-triggered mode where nodes start pumping video frames as soon as they detect an event and continue to do so as long as the target is within the sensing radius and the Field of View (FoV). The number of frames triggered by an event is variable and as shown in Fig. 1, it is a function of the duration of the event and the camera frame rate. Event duration is actually the target residence time inside the coverage area, which in turn depends on the target speed, $V$, and the path length, $D_{A B}$, covered inside the FoV.

The data traffic created by an individual node upon detection will be termed as an event-flow. An event-flow is identified as the sequence of image frames produced by the same source node triggered by the detection of a target. As opposed to the time-triggered (periodic) traffic pattern, for event-triggered traffic, the number of events created per unit time may easily reach high values depending on the number and mobility of the target(s). When combined with the large video frame sizes, this leads to instantaneous traffic volumes that exceeds the capacity of the network, which in turn results in packet drops due to buffer overflow. Our aim in this work is twofold. Firstly, we want to maximize the overall visual information content conveyed to the sink in the presence of packet drops. Secondly, we aim at reducing the delay experienced by the initial frames of an event. To achieve these, we introduce an application level fairness scheme called Event Based Fairness (EBF). Here, we identify event flows as the application level entities and we seek their fair treatment in the network. By distributing network resources equally among event-flows, we aim at lowering the impact of packet drops on the visual information carried. We provide two implementations of EBF, namely, Round Robin Fair Queueing based EBF (EBF-RR), and Least Attained Service (LAS) scheduling based EBF (EBF-LAS). In the simulation experiments, both EBF-RR and EBF-LAS are shown to perform better than the FCFS queueing where frames are serviced in their order of arrival. EBF-RR operates on the snapshot of the queue, hence, provides fairness among flows whose frames currently exist in the queue. Event-flows in a VSSN show an intermittent behavior, therefore, steady state flow rates may not always be attained. In this respect, EBF-LAS, which considers not only the current queue content but also 
the service history of the event-flows, is more successful in dealing with shortlived event-flows, hence provides better event level fairness. Operationally, when event-flows have to be handled simultaneously by a sensor node, EBF-LAS gives priority to the flow which has sent the least number of frames so far. This has two implications: (i) when a packet is dropped due to overflow, it is guaranteed to belong to a flow that has sent the maximum number of frames so far, i.e. the drop will decrease the information content of a flow that already transmitted the maximum visual information, (ii) in cases when no overflow is experienced, the frames of an event which has the smallest sequence number will have priority over frames of other event flows. Therefore, the delay of the initial frames will be decreased.

The rest of this paper is organized as follows: Section 2 gives an overview of the existing fairness approaches in both wired and wireless context. The motivation behind the EBF scheme is presented in Section 3. Internals of Event Based Fairness is discussed in Section 4. Experiment setup and results obtained are presented in Section 5. Finally, Section [6] concludes the paper.

\section{Related Work}

Fairness is well-studied in the context of wired networks 345]. For wireless communication, fairness is generally discussed according to the OSI level that fairness is supported. For instance, authors of [6] advocates that MAC level fairness alone cannot ensure the fairness of the whole wireless network, although MAC support can increase the efficiency for the fairness provided at the network layer. An option to make the network independent of the fairness issues at lower layers is to achieve fairness at the transport flow layer. A centralized max-min fairness approach for wireless mesh networks (WMN) which strives to achieve end-to-end fairness at the transport layer is presented in 7]. The centralized solution discussed is justified for WMNs but it is not applicable for the WSN case whether it be a scalar or a video based WSN. There are studies that specifically address fairness in WSNs 8910, among which Rangwala et al. proposes IFRC that combines fair bandwidth allocation with rate control [8]. In [1], feedback based congestion control mechanisms to enhance data delivery such as ESRT, CODA and SPEED 121314 are classified as reactive and the authors come up with a collision-free scheduling that provides max-min fairness in a proactive and distributed manner. In that sense, our work also can be characterized as proactive. A similar work by Tassiulas et al. proposes a scheduling scheme which achieves max-min fairness without giving the implementation level details of the MAC protocol [15].

As a transport protocol, ESRT [12 tries to carry the optimum number of packets from an event with a feedback mechanism from the sink to the nodes. However, the effectiveness of ESRT depends on the length of decision intervals $(\approx 10 \mathrm{sec})$ and the feedback latency. If the duration of the event is short as in surveillance applications and the feedback latency is high (e.g., the network diameter is high), the notification may arrive to the source after the end of the 
event and cannot avoid congestion. Moreover, ESRT is not designed to decrease the reporting delay of the events.

As mentioned in a recent survey on Least-Attained Service (LAS) [16, the Shortest Remaining Processing Time (SRPT) is optimal for minimizing the mean response time. SRPT gives precedence to the jobs with the shortest remaining time left by assuming that the queue dispatcher is aware of the residual size of the job that has not arrived yet. However in blind systems as in WSNs, although the job size may not be known, a job's age is always known, therefore instead of SRPT, a more practical policy, LAS scheduling is a better choice. In the literature, LAS scheduling exists in different names, such as Foreground-Background and Shortest-Elapsed-Time [16. Among them, the performance of LAS with respect to the variability of the job size is analyzed in [17. Authors indicate that while $99 \%$ of the jobs encounter a reduced conditional mean slowdown under LAS, less than $1 \%$ of the largest jobs experience a negligible increase of their conditional mean slow down. In [18, Wierman et al. showed that LAS outperforms Processor-Sharing with respect to the mean response time and the mean slowdown when the job size distribution has a decreasing failure rate. Furthermore Wierman et al. presents a classification of scheduling policies considering the unfairness in 19. Furthermore, the effect of LAS on heavy-tailed traffic in wireless networks is presented in [20. The authors compare LAS with RoundRobin (RR) based scheduling and show that LAS outperforms RR in a single bottleneck link and also in a one hop wireless shared link.

\section{Motivation}

When we focus on the contents of an event-flow, we observe that there is spatiotemporal redundancy among consecutive frames. This is mainly because the camera module of the sensor node takes continuous snapshots of the scene with a certain frame rate. It is not possible to generically define the number of frames to be received at the sink for healthy reception of the event. This depends on the type of detection method run on the back end. This could range from simple event detection in which only the existence of the event is notified to the classification or the identification of the target. Also the frames received could be an input to an image recognition engine or to an human operator. Another factor is the specific positioning and movement of the target within the visual sensing range. A target closer to the camera module takes a bigger portion of the picture, however assuming that the target is mobile, proximity to the sensor also implies shorter residence time inside the sensing range, hence a shorter event-flow. Therefore, we can crudely conclude that event-flows as they become longer, they contain more frames of the scene and likely to have more redundancy among frames. The information contribution of the individual frames of a generic event-flow is depicted in Fig. 2.

With this observation in mind, we propose that irrespective of the duration of the events, initial frames of an event deserve special care. That is because they contain much of the visual information and also the delay experienced by them 


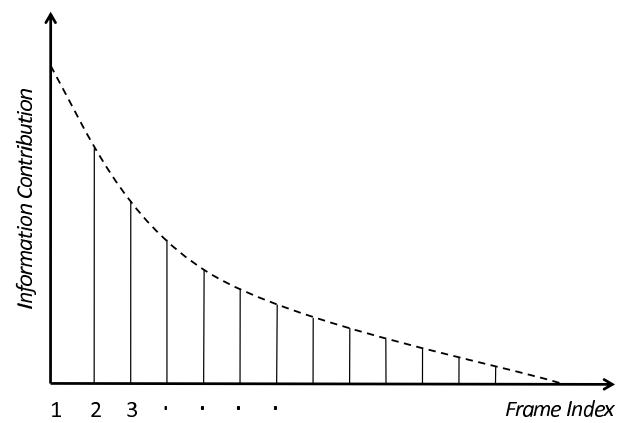

Fig. 2. Information contribution of individual frames of a surveillance video event-flow

directly affects the reporting delay. In this work, we give priority to the initial frames via an application level fair queue management scheme, namely Event Based Fairness.

\section{Providing Application Level Fairness with EBF}

WSNs, including VSSNs, have the unique characteristic that the network is designed to achieve a common specific task, in which all the nodes operate collaboratively. Opposingly, in the previous wired and wireless networking paradigms, we can see the clear distinction between the applications running on the nodes and the communication service provided by the network. In this picture, the nodes care about maximizing their own utility and not necessarily respecting the network wide resource scarcity. Therefore, previous studies on the fairness mainly focused on the per-node and the per-flow based fairness. For a WSN, on the other hand, the individual nodes may not need to obtain fair service at all times. However, fairness becomes a crucial issue when considered in terms of the application performance.

The performance of a VSSN application depends on how well the events are reported, i.e. the video quality of the events conveyed and the initial reporting delay which are related to how events are handled and processed in the network. In the standard FCFS queueing approach, the frames of the events are queued in the nodes according to the their sequence of arrival. A burst event with many frames fills the head of the queue in the relay node and other events can only utilize the space left from the former event. The rest of the events have to wait till the frames of the previous events are served. Moreover, in the case of buffer overflow, only a few of the packets of the forthcoming events can be relayed. As a result, when FCFS is preferred, while some events are reported with high quality and low latency, others are received in low quality and high latencies. In order to guarantee a certain Quality of Service (QoS) in video quality and acceptable reporting latency, we propose an event-aware fair queue management scheme called EBF that is streamlined for VSSN applications. 


\subsection{Round Robin Based Implementation of EBF}

Round Robin based EBF implementation (EBF-RR) strives to give fair service to all events that are currently enqueued in a VSSN node. The queue is composed of frames received from the network for relaying purposes and the frames received from the application layer, i.e. the video frames produced by the node itself. EBF$\mathrm{RR}$ operates by servicing frames of events in a round robin manner, one frame from each event at a time. Internally, EBF-RR dynamically forms logical queues for each event and gives service to each queue in a time-shared manner. The duration in which all event queues are served once is called an epoch. During an epoch, the available bandwidth is equally divided among each event. The overall service rate an event gets from EBF-RR depends on the length of the event (in terms of frames) occupied in the main queue, the total number of events in the queue, the length of each event in the queue and the congestion level experienced at the MAC level (available effective bandwidth). When the incoming frames are more than the capacity of the node, buffer overflow occurs. In that case, EBF-RR drops the frame from the longest event queue. With this scheme a received frame that arrives at the full main buffer need not be dropped unless it belongs to the event that currently has the longest logical queue. When compared with the FCFS behavior, EBF-RR provides fair bandwidth allocation to events and also gives priority to events with fewer frames. This latter property is especially more pronounced in the case of buffer overflows in which frames of events with longer queues are dropped. In that sense, EBF-RR tries to homogenize the service rate among events according to the snapshot of the queue.

One point to note in the above discussion is that the queue manipulation is done in terms of frames and not packets. Therefore in our VSSN implementation, SMAC [21] with Message Passing feature is used as the MAC layer. Since Message Passing allows frames to be passed among nodes intact which makes our assumption about frame based queue manipulation possible.

\subsection{LAS Based Implementation of EBF}

The main idea behind LAS Based EBF (EBF-LAS) is that an event is a sequence of frames flowing in the network and at a specific time instance, only a portion of it may be contained in the buffer of a VSSN node. This is due to the buffer size limitations and earlier frame drops that an event may experience. EBF$\mathrm{RR}$ operates on the instantaneous snapshot of the buffer and provide fairness among events according to what is currently present. In this respect, a way to provide better fairness among events is to consider not only the current buffer composition but also to take into account the frames of an event that has been relayed previously. EBF-LAS, like EBF-RR, forms logical queues of frames per event and service one frame from each queue in an epoch in a round robin fashion. However, unlike the RR implementation, EBF-LAS keeps track of the sent frames and inserts a virtual frame to the event queues as place holders for each frame of an event that is relayed. Therefore, a logical queue for an event contains both real frames that are waiting to be send and virtual frames that 


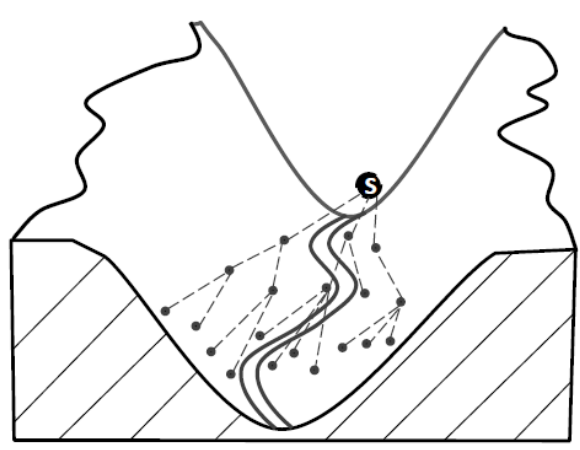

(a) Trespassers' Favorite Path (TFP)

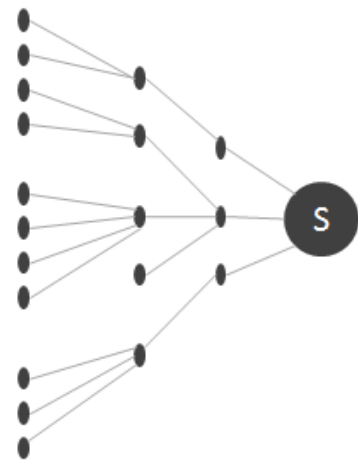

(b) Detailed view of the TFP multi-hop simulation scenario

Fig. 3. Deployment Scenario. Intruders follow the favorite path in which the sensors are deployed more densely.

are already sent. In every logical event queue, virtual frames are placed in the front of the queue, therefore, when deciding on the next frame to get relayed, EBF-LAS gives explicit priority to the events that have fewer frames sent.

\section{Comparative Evaluation of EBF-RR, EBF-LAS and FCFS}

\subsection{Experimental Setup}

We examine the effect of EBF-RR and EBF-LAS using the OPNET simulation environment 22]. In order to observe the improvements on the reporting latency and the video quality of events in detail, a surveillance scenario is examined. In a geographical area that is under surveillance not every path is equally likely to be used. The paths which intruders have higher tendency to follow are called Trespassers' Favorite Path (TFP) 23. TFPs are preferred over other alternatives due to the reasons such as easy geographical conditions and remoteness to checkpoint locations. For effective operation, existence of TFPs should be considered when designing a surveillance network, as large portion of the total traffic is likely to be originated from sensor nodes located within the TFPs. In our surveillance scenario, we have simulated the traffic created in a valley-type TFP which contains 19 VSSN nodes, as depicted in Fig. 3 ,

In video surveillance applications the volume of the data traffic is related to the dwell time of the target, the camera frame rate and the compression algorithm. The duration of an event and the number of frames created during the event varies according to these parameters. In our tests, we model the traffic creation using event size, $\Psi$, frame interarrival time, $\Delta_{F}$, and event interarrival time, $\Delta_{E}$. $\Psi$ denotes the number of frames contained in an event, whereas $\Delta_{F}$ is the frame 
Table 1. Simulation Parameters

\begin{tabular}{ll}
\hline \hline Parameter & Value \\
\hline \hline Event Size $(\Psi)$ & Normal distributed with $\left(\mu=15, \sigma^{2}=7\right)$ Frames \\
\hline Video Frame Size & 10 Kbits \\
\hline Packet Size & 1 KBits \\
\hline Frame Interarrival Time $\left(\Delta_{F}\right)$ & Uniformly distributed with $\mu=1 / 3 \mathrm{sec}$ \\
\hline Event Interarrival Time $\left(\Delta_{E}\right)$ & Exponentially distributed with $\mu=25 \mathrm{sec}$ \\
\hline Duty Cycle & $10 \%, 20 \%^{*}, 30 \%, 40 \%$ \\
\hline Bandwidth & $250 \mathrm{Kbps}$ \\
\hline Buffer size & $100 \mathrm{Kbits}$ \\
\hline MAC layer & SMAC $[21]$ with Message Passing feature \\
\hline Number of Repetitions & 20 \\
\hline Confidence Interval & $95 \%$ \\
\hline
\end{tabular}

(*)Unless otherwise specified, $20 \%$ duty cycle is the default in the experiments.

generation rate of the camera modules of the VSSN nodes. $\Delta_{E}$ models the time between two consecutive events a sensor node detects. Values for $\Psi, \Delta_{F}, \Delta_{E}$ and other related simulation parameters are presented in Table 1 .

Preliminary experiments are run to fix the buffer capacity that is allocated on the individual nodes. Enlarging the buffer enhances the throughput at the expense of increased delay. After a threshold value, larger buffer sizes result in intolerable delay levels [24]. The chosen buffer size (100 Kbits) is within an operationally feasible region in which the delay-throughput balance is observed. Please refer to 25] for a detailed study in which the effects of the factors like buffer size, camera frame rate and MAC duty cycle on the VSN performance are systematically examined.

\subsection{Results}

A histogram summarizing the events according to the number of successfully received frames at the sink is presented in Fig. 4(a). Out of the 2220 events generated, for the FCFS case, for instance, around 130 events are reported only with a single frame whereas around 90 events are reported with 14 frames. The number of events reported for all queueing mechanisms are close to each other 2122, 2215 and 2211 for FCFS, EBF-RR and EBF-LAS respectively. However, it is observed that the variance in the frequency of the frames per event is decreased by EBF-RR and EBF-LAS. In the $1-3$ fps interval and $10-20$ fps interval, the number of events are less in EBF-RR and EBF-LAS cases than FCFS since EBF-LAS and EBF-RR decreases the number of over reported events and share the available excess bandwidth among the under reported events. Thus, most of the events are reported similarly which is due to the fair treatment of frames according to the related events.

The total number of frames required to be received at the sink in order a triggered event to be considered as detected depends on the application. However, 


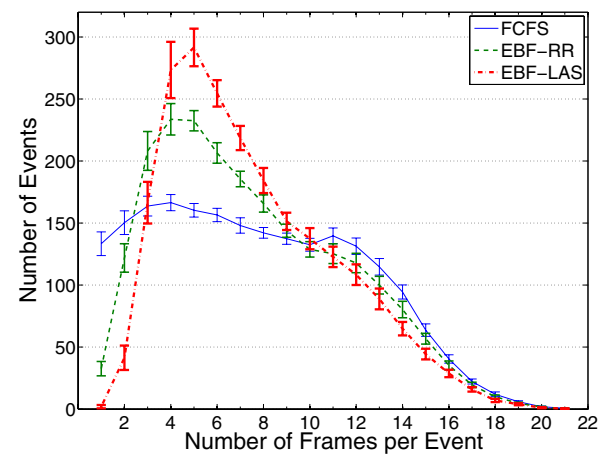

(a) Histogram of the number of frames received at the sink for each event.

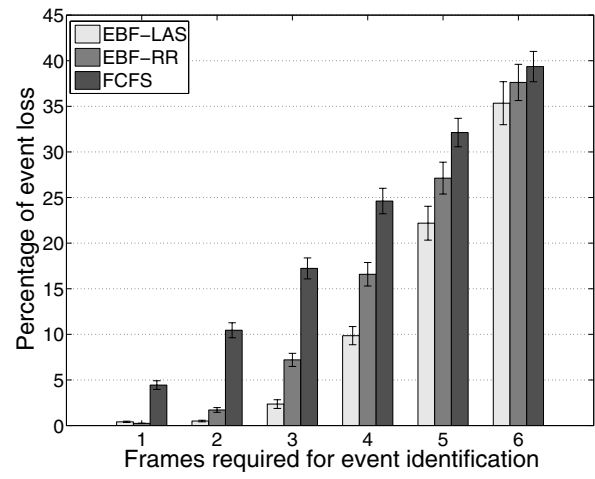

(b) Missed event ratios with different number of frames required for the identification.

Fig. 4. Histogram and miss ratio for events which are composed of variable number of frames

as previously pointed out, it is the initial frames of an event that contribute more to the visual information received at sink. In order to observe the effect of EBF-LAS and EBF-RR on the reliable event reporting, we plot the ratio of missed events in Fig. 4(b). As expected, when the required frames per event is increased, missed event ratio also increases in all queueing techniques. However, the ratio of missed events are clearly less in EBF-RR and EBF-LAS cases compared to that of the FCFS case. For instance, when the required frames for event identification is set to 4, while FCFS misses $24 \%$ of the events, EBF-RR misses $17 \%$ and EBF-LAS misses less than 10\%. Additionally, the difference between the FCFS, EBF-RR and EBF-LAS systems decreases as the number of required frames increases. The reason is that EBF-LAS punishes the large events while giving precedence to smaller ones especially when the network load becomes high.

Besides the video quality, the reporting latency of the events are also important. Especially the frame of an event which arrives first to the sink has the most significant contribution for the event reporting since it makes the sink aware of that event. Fig. 5(a) presents the average of the first frame latency of the events with various duty cycles and indicates that EBF-RR and EBF-LAS improve the event reporting delay significantly compared to the FCFS case.

To have a more general understanding of the latency behavior of the events, Fig. 5(b) depicts the average delay a certain frame of an event experiences, e.g., the average latency of the $8^{\text {th }}$ frame of the events. It is observed that EBFLAS decreases the delay for all frames of the events, whereas EBF-RR performs better than FCFS up until the $4^{\text {th }}$ frames of the events. In other words EBF-LAS decreases the mean response time of the events.

On the other hand, when the duty cycle is increased, most of the frames arrive to the sink. Therefore as in Fig. 6(a), the difference between the intelligent queueing techniques and the FCFS decreases. However, as observed in 6(b) 


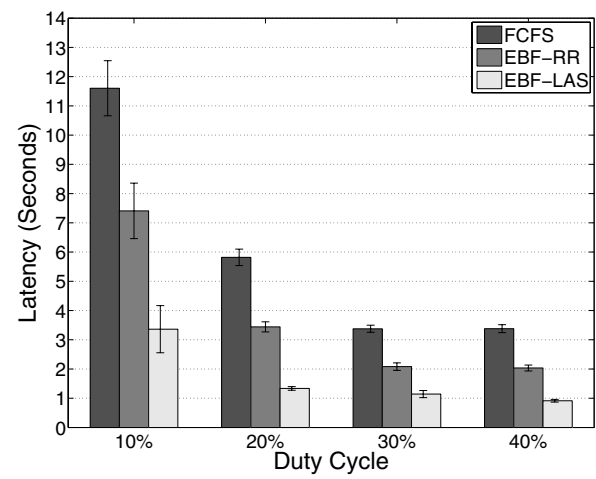

(a) Average delay for the first arriving frame of an event. (using various duty cycles)

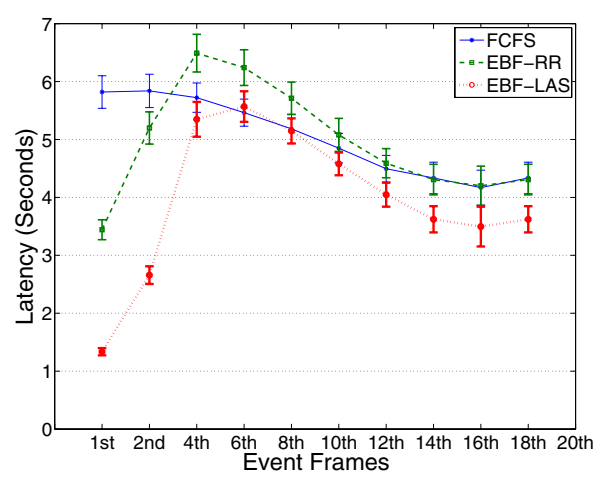

(b) Average latency of frames.

Fig. 5. Frame latencies of the events.(20\% default duty cycle)

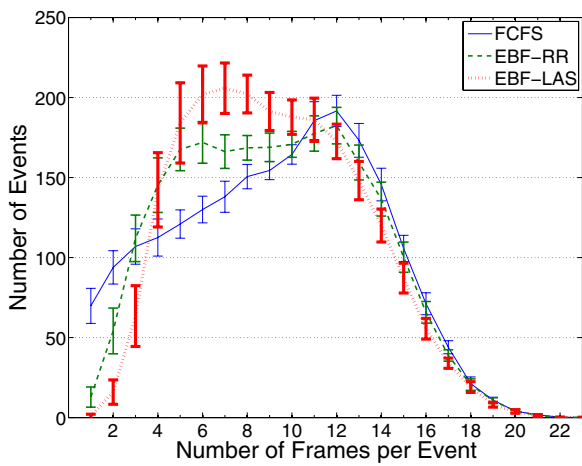

(a) Histogram of the number of frames reached to sink for each event

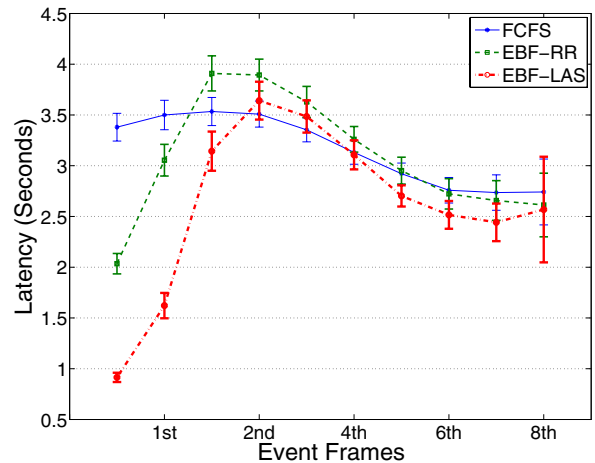

(b) Average latency of frames

Fig. 6. Latency and Histogram of the events. (40\% duty cycle).

compared with FCFS, EBF-RR and EBF-LAS can relay all frames without a significant sacrifice in terms of latency.

\section{Conclusions and Future Work}

In this work, Event Based Fairness (EBF) for Video Surveillance Sensor Networks (VSSNs) is introduced. Main goals of EBF are to increase the event reception ratio at the sink node and to decrease the initial reporting delay of the events. There is inherently high volume of traffic in a VSSN when an intruder(s) is present. Most of the time, the traffic produced is more than that can effectively be carried by the network. To be able to achieve its design goals, EBF defines 
an event, as opposed to a video frame or a network packet, to be the logical messaging unit in a VSSN and introduces the fair treatment of events which are application level entities. Two different EBF implementations, namely EBF-RR and EBF-LAS are compared with the legacy FCFS style queue management. $\mathrm{EBF}$ is shown to not only increase the number of events that are reported properly but also to lower the initial reporting delay considerably. As a future work, we plan to implement cross-layer solutions in which MAC level priority mechanisms are co-implemented with the EBF framework to further enhance VSSN functionality.

\section{References}

1. Downes, I., Rad, L., Aghajan, H.: Development of a mote for wireless image sensor networks. In: Proc. of COGnitive systems with Interactive Sensors (COGIS), Paris, France (March 2006)

2. Rahimi, M., Baer, R., Iroezi, O.I., Garcia, J.C., Warrior, J., Estrin, D., Srivastava, M.: Cyclops: in situ image sensing and interpretation in wireless sensor networks. In: SenSys 2005: Proceedings of the 3rd international conference on Embedded networked sensor systems, pp. 192-204. ACM Press, New York (2005)

3. Demers, A., Keshav, S., Shenker, S.: Analysis and simulation of a fair queueing algorithm. In: SIGCOMM 1989: Symposium proceedings on Communications architectures \& protocols, pp. 1-12. ACM, New York (1989)

4. Shreedhar, M., Varghese, G.: Efficient fair queueing using deficit round robin. SIGCOMM Comput. Commun. Rev. 25(4), 231-242 (1995)

5. Bertsekas, D., Gallager, R.: Data networks, 2nd edn. Prentice-Hall, Inc., NJ (1992)

6. Jun, J., Sichitiu, M.: Fairness and qos in multihop wireless networks. In: IEEE 58th Vehicular Technology Conference, VTC, vol. 5, pp. 2936-2940 (2003)

7. Raniwala, A., De, P., Sharma, S., Krishnan, R., cker Chiueh, T.: End-to-end flow fairness over ieee 802.11-based wireless mesh networks. In: IEEE INFOCOM, pp. 2361-2365 (2007)

8. Rangwala, S., Gummadi, R., Govindan, R., Psounis, K.: Interference-aware fair rate control in wireless sensor networks. In: Rizzo, L., Anderson, T.E., McKeown, N. (eds.) SIGCOMM, pp. 63-74. ACM, New York (2006)

9. Fan, K.-W., Zheng, Z., Sinha, P.: Steady and fair rate allocation for rechargeable sensors in perpetual sensor networks. In: Proc. of ACM SENSYS, Raleigh, NC (November 2008)

10. Chen, S., Fang, Y., Xia, Y.: Lexicographic Maxmin Fairness for Data Collection in Wireless Sensor Networks. IEEE Transactions On Mobile Computing, 762-776 (2007)

11. Sridharan, A., Krishnamachari, B.: Max-min fair collision-free scheduling for wireless sensor networks. In: Workshop on Multihop Wireless Networks (MWN 2004), IPCCC (2004)

12. Akan, O.B., Akyildiz, I.F.: Event-to-sink reliable transport in wireless sensor networks. IEEE/ACM Trans. Netw. 13(5), 1003-1016 (2005)

13. Wan, C.-Y., Eisenman, S.B., Campbell, A.T.: Coda: congestion detection and avoidance in sensor networks. In: SenSys 2003: Proceedings of the 1st international conference on Embedded networked sensor systems, pp. 266-279. ACM, New York (2003) 
14. He, T., Stankovic, J.A., Lu, C., Abdelzaher, T.F.: Speed: A stateless protocol for real-time communication in sensor networks. In: ICDCS, pp. 46-55 (2003)

15. Tassiulas, L., Sarkar, S.: Maxmin fair scheduling in wireless networks. In: INFOCOM (2002)

16. Nuyens, M., Wierman, A.: The Foreground-Background queue: A survey. Performance Evaluation 65(3-4), 286-307 (2008)

17. Rai, I., Urvoy-Keller, G., Biersack, E.: Analysis of LAS scheduling for job size distributions with high variance. In: Proceedings of the 2003 ACM SIGMETRICS international conference on Measurement and modeling of computer systems, pp. 218-228. ACM Press, New York (2003)

18. Wierman, A., Bansal, N., Harchol-Balter, M.: A note on comparing response times in the $\mathrm{M} / \mathrm{GI} / 1 / \mathrm{FB}$ and $\mathrm{M} / \mathrm{GI} / 1 / \mathrm{PS}$ queues. Operations Research Letters 32(1), 73-76 (2004)

19. Wierman, A., Harchol-Balter, M.: Classifying scheduling policies with respect to unfairness in an M/GI/1. ACM SIGMETRICS Performance Evaluation Review 31(1), 238-249 (2003)

20. Shao, Z., Madhow, U.: Scheduling heavy-tailed data traffic over the wireless Internet. In: Proceedings of IEEE 56th Vehicular Technology Conference, 2002. VTC 2002-Fall, vol. 2 (2002)

21. Ye, W., Heidemann, J., Estrin, D.: Medium access control with coordinated adaptive sleeping for wireless sensor networks. IEEE/ACM Trans. Netw. 12(3), 493-506 (2004)

22. Inc, O.: OPNET Modeler

23. Komar, C., Ersoy, C.: Optimization of power consumption using trespassers favorite path and variable sensing range integrated sleep schedule in surveillance wireless sensor networks. In: 23rd International Symposium on Computer and Information Sciences, 2008. ISCIS 2008, pp. 1-5 (2008)

24. Ozgovde, A., Demirkol, I., Ersoy, C.: Effect of sleep schedule and frame rate on the capabilities of Video Sensor Networks. In: 3rd International Symposium on Wireless Pervasive Computing, 2008. ISWPC 2008, pp. 156-159 (2008)

25. Durmus, Y., Ozgovde, A., Ersoy, C.: Exploring the effect of the network parameters of video sensor networks. In: Proceedings of ISCN 2008 8th International Symposium on Computer Networks, pp. 188-192 (2008) 